\title{
Effects of ketogenic diet containing medium-chain fatty acids on serum inflammatory factor and mTOR signaling pathway in rats
}

\author{
Huan Liu ${ }^{1}$, Jingwei Huang ${ }^{1}$, Hui Liư ${ }^{2}$, Feng Li ${ }^{3}$, Quansheng Peng ${ }^{4^{*}}$ and Chunhong Liu ${ }^{{ }^{*}}$
}

\begin{abstract}
Background: The ketogenic diet (KD) can promote the anti-inflammatory metabolic state and increase ketone body level in rats. This study was to explore the effects and differences of KD with or without medium-chain fatty acids (MCFAs) on serum inflammatory factors and mTOR pathway in Sprague-Dawley (SD) rats.

Results: Male SD rats were assigned to five groups: control diet (C), 20\% caloric restriction diet (LC), 20\% caloric restriction ketogenic diet (containing MCFAs) (LCKD1), 20\% caloric restriction ketogenic diet (LCKD2) and 20\% caloric restriction foreign ketogenic diet (LCKD3), and fed for $30 \mathrm{~d}$. LC and KD could significantly reduce the body weight of rats; $L C$ and KD containing MCFAs showed anti-inflammatory effects; $K D$ without MCFAs decreased the concentration of mTOR1, while KD containing MCFAs decreased the expression of AMPK, mtor1 and P70sk.
\end{abstract}

Conclusions: KD containing MCFAs showed better effects on the mTOR pathway and anti-inflammation than that without MCFAs.

Keywords: Ketogenic diet, Medium-chain fatty acids, mTOR, Weight, Anti-inflammatory

\section{Background}

Ketogenic diet (KD) is a special diet formula that consists of high fat, appropriate protein, low carbohydrate and appropriate other nutritional elements [1-3]. As a special diet, it can improve the incidence of epilepsy. It uses fat instead of glucose as the energy source to increase the efficiency of ketone production and protects nerves from damage. The KD mimics a hungry state, but with different metabolic patterns. Under normal conditions, the body's energy supply mainly comes from glucose oxidative phosphorylation pathway, but when the carbohydrate intake was insufficient (such

\footnotetext{
*Correspondence: quanshp@scau.edu.cn; liuch@scau.edu.cn

${ }^{1}$ College of Food Science, Guangdong Provincial Key Laboratory

of Food Quality and Safety, South China Agricultural University,

Guangzhou 510642, China

${ }^{4}$ Logistics Department, South China Agricultural University,

Guangzhou 510642, China

Full list of author information is available at the end of the article
}

as hunger, high fat diet), fat metabolism becomes the main energy source. In the process of fat metabolism, fatty acids are first $\beta$-oxidized into ketones in the liver, producing multiple metabolites including acetoacetate (acetoacetate), $\beta$-hydroxy butyric acid ( $\beta$-hydroxy butyrate) and pyruvate (acetone), which are then transported to tissues and organs used as energy source when lack of glucose. Since the main energy-supplying substances are changed from carbohydrates to fats, the KD induces physiological ketonemia in the body [4], which may improve the incidence of epilepsy.

The KD treatment can be traced back to 1921, when Dr. Wilder of the Mayo Clinic applied KD in the treatment of intractable epilepsy in children [5]. Since then, a number of large-scale follow-up studies have been carried out, which have confirmed that KD has a good therapeutic effect on epilepsy [6-8]. In recent decades, KD has once again attracted much attention due to its 
potential application in metabolic defect diseases, for instance, pyruvate dehydrogenase, psychiatric illnesses such as bipolar disorder, depression, neural degenerative diseases such as Parkinson's and Alzheimer's disease, malignant tumors such as prostate cancer, etc. [9].

KD using medium-chain triglycerides (MCT) instead of fat sources was introduced in the 1970s [10]. It only provides about $45 \%$ of dietary energy when compared with the classic KD [11]. MCT are directly absorbed from the portal vein in the form of free fatty acids, thus they reach the liver faster than long-chain fatty acids (LCT, 14 carbon) that are absorbed from the small intestine and the lymphatic system. Besides, the $\beta$-oxidation of MCT in the liver is rapid because it does not need to be activated by coenzyme A, making MCT a good ketogenic substrate. MCT KD is widely used in the treatment of drug-resistant epilepsy worldwide, mainly in children [12], but also in adults. Additionally, several studies have observed direct actions on seizure activity and mitochondrial function when mediumchain fatty acids (MCFAs) were provided in the MCT KD [13-15].

mTOR, a member of the phosphatidyl-inositol kinaserelated protein kinase family, is an important regulator for the nutrients availability in mammals, and is a key mediator of cell growth mechanism signaled by insulin, insulin-like growth factor 1 and other growth-factor signals $[16,17]$. mTOR could be activated by phosphoinositide-3 kinase (pi3k)/Akt signal in the presence of nutrients and growth factors, yet could be inhibited by AMP-activated protein kinase (AMPK) in the absence of energy. mTOR is the key node to control cell growth and proliferation by regulating the translation of S6Ks and 4e-bp1 [18]. Recent studies showed that activation phosphorylation of S6K and inactivation phosphorylation of 4E-BP1 were related to PI3K-induced tumorigenesis [19]. Therefore, mTOR signaling pathway could be a suitable candidate for understanding the mechanism of KD modulation, due to its ability to integrate nutrient and energy signals.

The aim of the present study was to explore the effects of KD on serum hormones, ketone bodies, blood sugar, inflammatory factors and mTOR signaling pathways in rat fed with ketogenic diet containing MCFAs.

\section{Materials and methods}

\section{Treatment of animals}

All experiments were approved by the Animal Care Committee of the laboratory Animal Center of South China Agricultural University, Guangzhou. During 7 days of adaptive feeding period, adult male Sprague-Dawley rats (SYXK2014-0136) weighing 140-160 g, were housed under controlled conditions (12 h light/dark cycle,
$22 \pm 0.5^{\circ} \mathrm{C}$ and $50-60 \%$ relative humidity), and fed with a rodent chow and tap water ad libitum. Then the rats were randomly divided into five groups with ten animals in each group (C: Control diet; LC: $20 \%$ caloric restriction diet; LCKD1: 20\% caloric restriction ketogenic diet (fat: protein + carbohydrate $=4: 1$, containing 30\% MCFA of total fat); LCKD2: 20\% caloric restriction ketogenic diet, fat: protein + carbohydrate $=4: 1$; LCKD3: $20 \%$ caloric restriction foreign ketogenic diet, fat: protein + carbohydrate $=4: 1)(K D 1$ and KD2 were provided by Guangzhou ketone Co. Ltd; KD3 were bought from NUTRICIA, France) (the daily intake of LC, LCKD1, LCKD2 and LCKD3 groups was adjusted to $80 \%$ of control group). The rats were weighed every 5 days, and before the subacute experiment at 30 days, they were fasted for $12 \mathrm{~h}$, and then killed by cervical dislocation. Serums were obtained by centrifuging the blood samples at $845 \mathrm{~g}$ at $4{ }^{\circ} \mathrm{C}$ for $15 \mathrm{~min}$, and then stored at $-80^{\circ} \mathrm{C}$ until use. The livers, kidneys and testes were carefully separated with ophthalmic tweezers and surgical scissors. Adipose tissue and connective tissue around the organs were removed as much as possible. After separation, the livers, kidneys and testes were, respectively, washed with pre-cooled saline, and then weighed after suction with absorbent paper. Low temperature was maintained throughout the dissection. The organ coefficient was expressed as the ratio of organ weight $(\mathrm{g})$ to body weight $(\mathrm{g})$. All animal procedures were performed according to the guidelines from Directive 2010/63/EU of the European Parliament and the National Institutes of Health with the purpose of protection of animals used for scientific purposes.

\section{Detection of blood ketone}

D-3-Hydroxybutyric acid in blood was catalyzed to acetoacetic acid by D-3-hydroxybutyric acid dehydrogenase, immobilized on the surface of the test strip, during which NAD was reduced to NADH. A constant working voltage applied to the test strip oxidized $\mathrm{NADH}$ to NAD, resulting in an oxidation current. The magnitude of the oxidation current was proportional to the concentration of D-3-hydroxybutyric acid, so the content of D-3-hydroxybutyric acid can be calculated according to the magnitude of oxidation current in the tester.

\section{Determination of serum hormone, blood sugar and serum inflammatory factors levels}

Concentrations of insulin, adiponectin, blood sugar, hs-CPR, TNF- $\alpha$, IL- 6 , and ENA-78 were determined by ELISA kits from Jiang Lai (Shanghai, China) using double antibody sandwich assay, according to the manufacturer's instructions. During the determination, the purified rat antibody (insulin, adiponectin, blood 
sugar, hs-CPR, TNF- $\alpha$, IL-6, and ENA-78, individually in the kit) on the surface of the solid carrier only reacted with the corresponding antibody in the sample (insulin, adiponectin, blood sugar, hs-CPR, TNF- $\alpha$, IL- 6 , and ENA-78). After adding enzyme-labeled antigen or antibody, the substrate was catalyzed by enzyme to a colored product. The sample content was directly related to the amount of the substance tested in the standard. The concentrations of insulin, adiponectin, blood sugar, hs-CPR, TNF- $\alpha$, IL- 6 , and ENA-78 in the samples were then calculated by comparing the O.D. to the standard curve.

\section{Determination of serum mTOR signaling pathway}

Concentrations of serum AMPK, mTOR, mTOR1, Pi3K, p70sk, 4e-bp, Beclin-1 and LC-3 were determined by using ELISA kits from Jiang Lai (Shanghai, China) according to the manufacturer's instructions. The test method is the same as 2.3 .

\section{Statistical analysis}

The SPSS (version 20.0) with one-way variance analysis was employed for statistical analysis of the results comparison among different groups. $P<0.05$ was considered as statistical difference.

\section{Result}

Effect of ketogenic diet on weight and serum hormone in rats

All groups gained body weight during the study period. The lowest weight gain was observed in LCKD1 group from day 11 to $30(P<0.05$; Fig. $1 \mathrm{c})$, and the highest weight gain was presented in control group from day 2 to $30(P<0.05$; Fig. 1 c). During day 8 to $17, \mathrm{LC}$ group exhibited a higher weight gain than LCKD1, LCKD2 and LCKD3 $(P<0.05$; Fig. 1c), but LCKD2 and LCKD3 did not significantly differ with LC from day 18 $(P>0.05$; Fig. 1c).
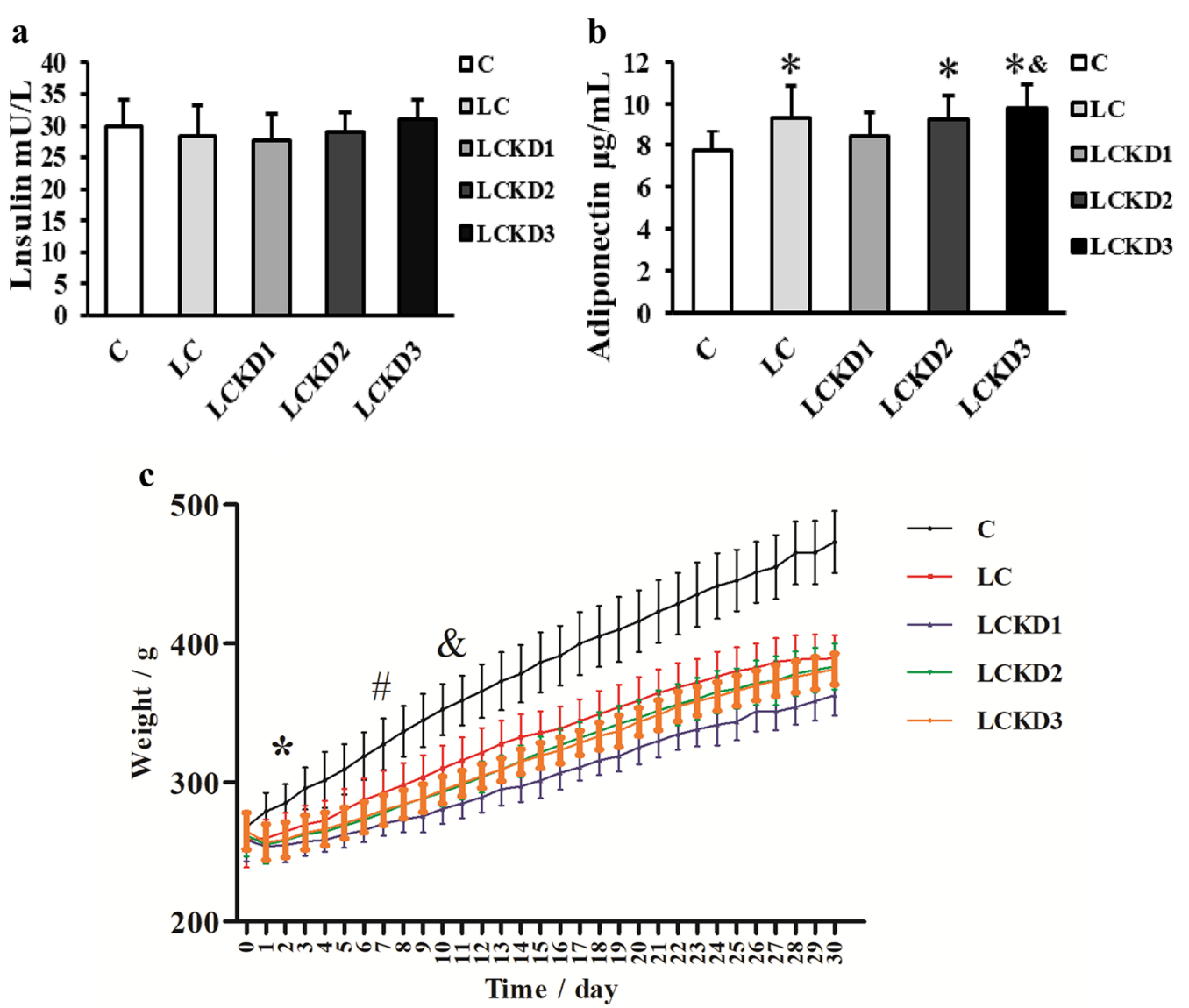

Fig. 1 Comparison of body weight, insulin and adiponectin levels in the rat serum in different groups. a The levels of insulin; $\mathbf{b}$ the levels of adiponectin; $\mathbf{c}$ body weight of the rats. Data are given as means \pm SD. Asterisks $\left(^{*}\right)$ represent significant differences from the control group ${ }^{*}$ $P<0.05$ ); octothorpe (\#) represents significant differences from the LC group ( $P<0.05$ ); ampersand $(\&)$ represents significant differences from the LCKD1 group $(\& P<0.05)$ 
Insulin and adiponectin levels in serum were determined in order to investigate the effects on serum hormones treated by ketogenic diet and medium-chain fatty acids. As shown in Fig. 1, insulin was not affected by ketogenic diet and medium-chain fatty acids diet $(P>0.05$; Fig. 1a). While compared with control group, LC, LCKD2 and LCKD3 groups increased the levels of adiponectin $(P<0.05$; Fig. $1 \mathrm{~b})$, and the adiponectin in LCKD3 group was higher than that in LCKD1 group $(P<0.05$; Fig. $1 \mathrm{~b})$.

\section{Effect of ketogenic diet on blood sugar and blood ketone in rats}

The effects of ketogenic diet and medium-chain fatty acids on blood sugar and blood ketone levels were explored by measuring the serum hormones. As shown in Fig. 2, LCKD3 showed higher blood sugar than control group $(P<0.05$; Fig. 2a). LCKD1, LCKD2 and LCKD3 significantly increased blood ketone, and LCKD3 was higher in blood ketone than LCKD1 $(P<0.05$; Fig. 2b).

\section{Effect of ketogenic diet on serum inflammatory factors in rats}

As shown in Fig. 3, TNF- $\alpha$ level was decreased in LC and LCKD1 groups $(P<0.05$; Fig. $3 \mathrm{a})$, and it was higher in LCKD2 and LCKD3 than that in LCKD1 $(P<0.05$; Fig. 3a). For hs-CPR content, it was higher in LCKD3 than that in LCKD1 $(P<0.05$; Fig. 3c). And ENA-78 was increased in LC when compared with control group $(P<0.05$; Fig. $3 \mathrm{~d})$. There was no obvious difference in IL-6 levels among the groups $(P>0.05$; Fig. $3 \mathrm{~b})$.
Effect of ketogenic diet on mTOR signaling pathway in rats Effect of ketogenic diet on upstream signaling pathway of serum mTOR in rats

AMPK and Pi3K as affected by KD and MCT are shown in Fig. 4, serum AMPK levels were significantly higher in the LCKD2 and LCKD3 groups compared with LCKD1 group $(P<0.05$; Fig. 4a), and serum AMPK levels were significantly lower in LCKD1 compared with C and LC groups $(P<0.01$; Fig. $4 a)$. But LCKD1 group showed higher serum Pi3K content when compared with LC group $(P<0.05$; Fig. 4b).

\section{Effect of ketogenic diet on serum mTOR and mTOR 1 in rats}

The serum mTOR and mTOR1 were measured at the end of treatments. As shown in Fig. 5, serum mTOR1 was significantly lower in LCKD1 and LCKD2 groups when compared with LC group $(P<0.05$; Fig. $5 b)$. But similar serum mTOR content was found in all the treatment groups $(P>0.05$; Fig. $5 \mathrm{a})$.

\section{Effect of ketogenic diet on downstream signaling pathway of serum mTOR and serum phagocytosis factor in rats}

The changes of downstream signaling pathway of serum mTOR and serum phagocytosis factor, as represented by P70sk, 4e-bp, beclin-1 and LC-3, are shown in Fig. 6 . Serum P70sk was significantly higher in LCKD1 than that in C, LC and LCKD3 groups $(P<0.05$; Fig. 6a). And serum beclin-1 in LCKD3 was higher than that in LCKD1 and LCKD2 groups $(P<0.05$; Fig. $6 \mathrm{c})$. However, the 4e-bp and LC-3 were at same level in all groups $(P>0.05$; Fig. 6b, d).
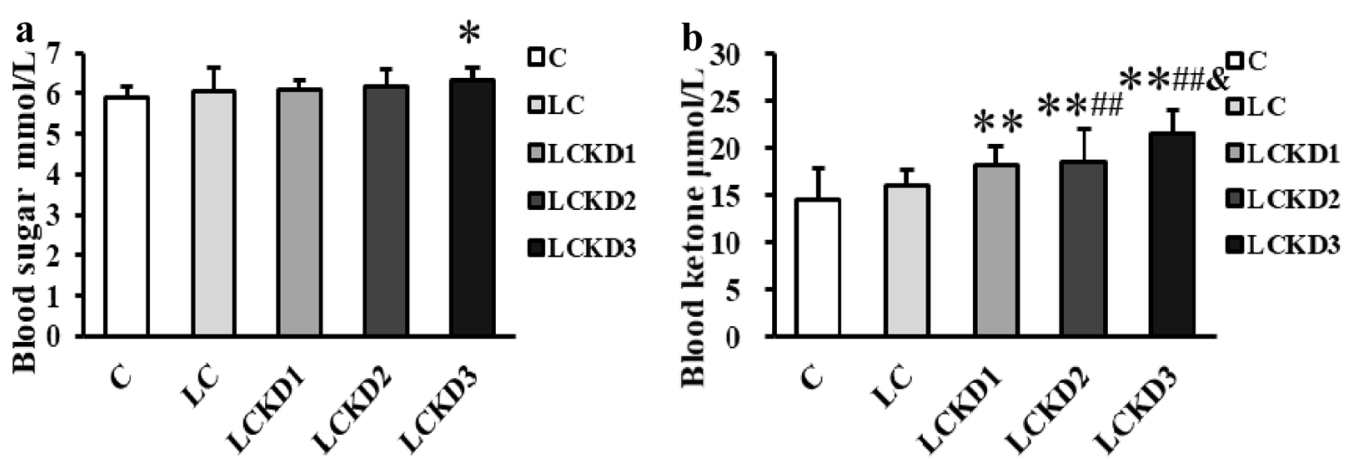

Fig. 2 Comparison of blood sugar and blood ketone levels in the rat serum in different groups. a The levels of blood sugar; $\mathbf{b}$ the levels of blood sugar. Data are given as means \pm SD. Asterisks $\left(^{*}\right)$ represent significant differences from the control group $\left({ }^{*} P<0.05 ; *^{*} P<0.01\right)$; octothorpe $\left(^{*}\right)$ represents significant differences from the LC group $\left({ }^{\# \#} P<0.01\right)$; ampersand $\left(^{\&}\right)$ represents significant differences from the LCKD1 group $\left({ }^{\&} P<0.05\right)$ 

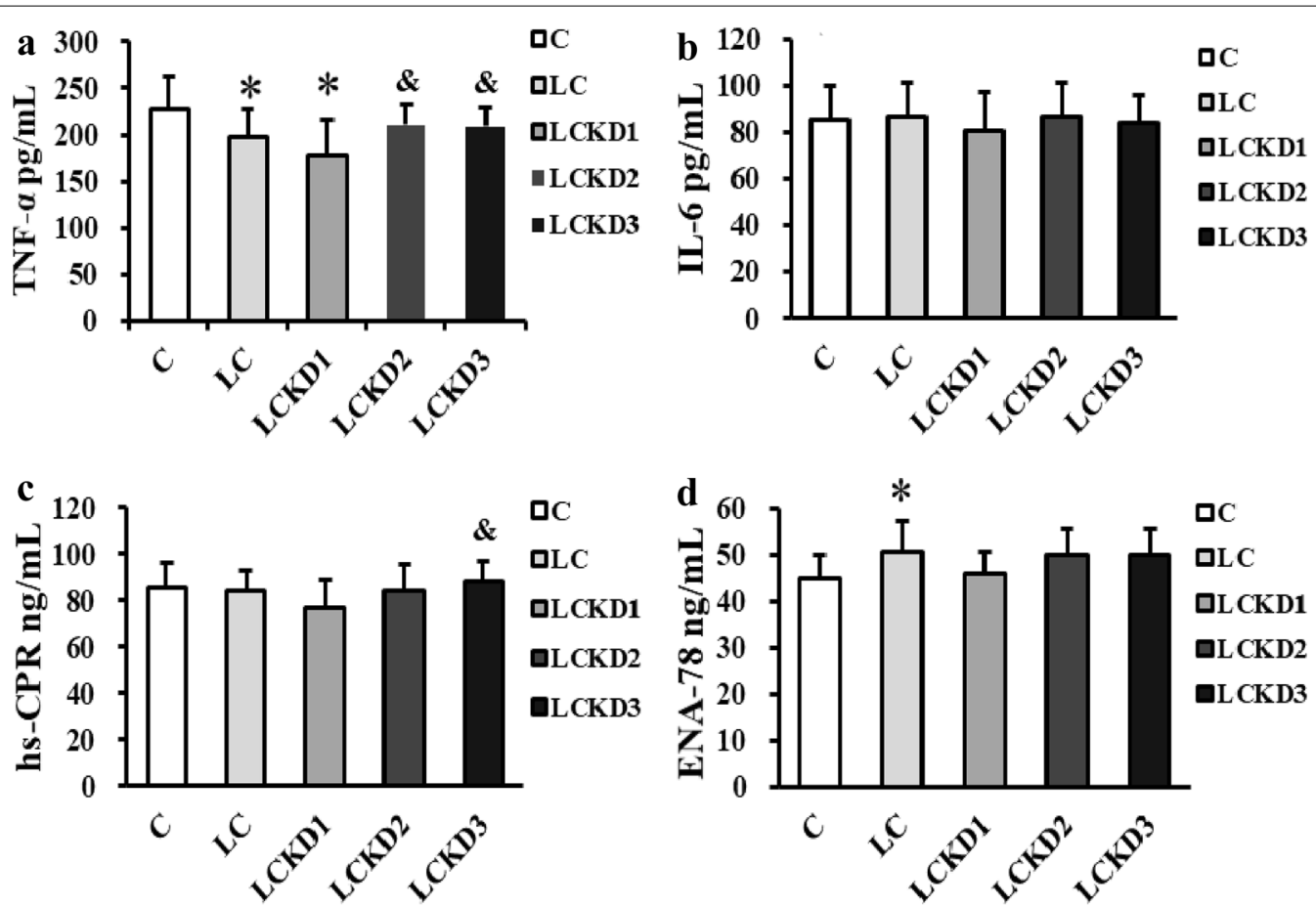

Fig. 3 Comparison of TNF-a, IL-6, hs-CPR and ENA-78 levels in the rat serum in different groups. a The levels of TNF-a; $\mathbf{b}$ the levels of IL-6; $\mathbf{c}$ the levels of hs-CPR; $\mathbf{d}$ the levels of ENA-78. Data are given as means \pm SD. Asterisks $\left(^{*}\right)$ represent significant differences from the control group $\left({ }^{*} P<0.05\right)$; ampersand $\left(^{\&}\right)$ represents significant differences from the LCKD1 group $(\& P<0.05)$
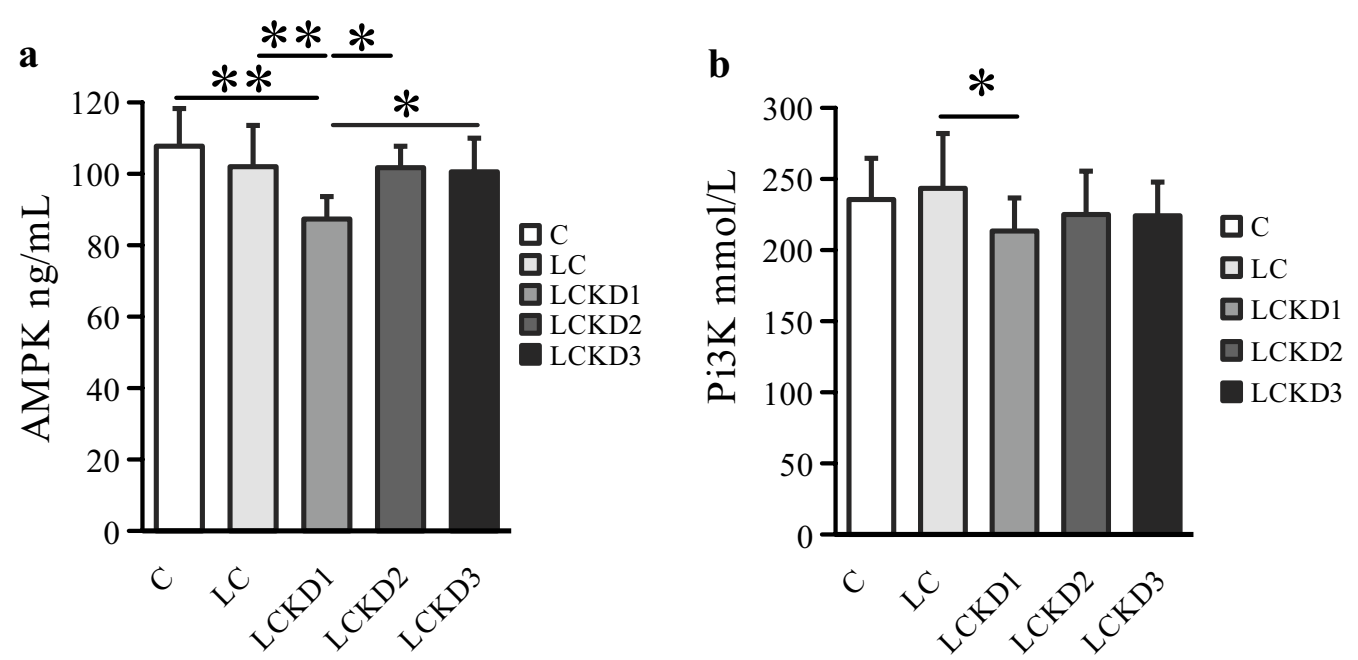

Fig. 4 Comparison of AMPK and Pi3K levels in the rat serum in different groups. a The levels of AMPK; $\mathbf{b}$ the levels of Pi3K. Data are given as means \pm SD. Asterisks ${ }^{*}$ ) represent significant differences between the two groups $\left({ }^{*} P<0.05 ;{ }^{*} P<0.01\right)$

\section{Discussion}

It is obvious from Fig. 1 that restriction diet and KD reduced the weight of rats. The finding was in accord with other studies, which found that rats in KD group consumed more calories than control group and distinct weight loss was observed after 1 week of KD administration [20]. Interestingly, compared with LC, significant weight loss was detected in LCKD2 and LCKD3 groups 

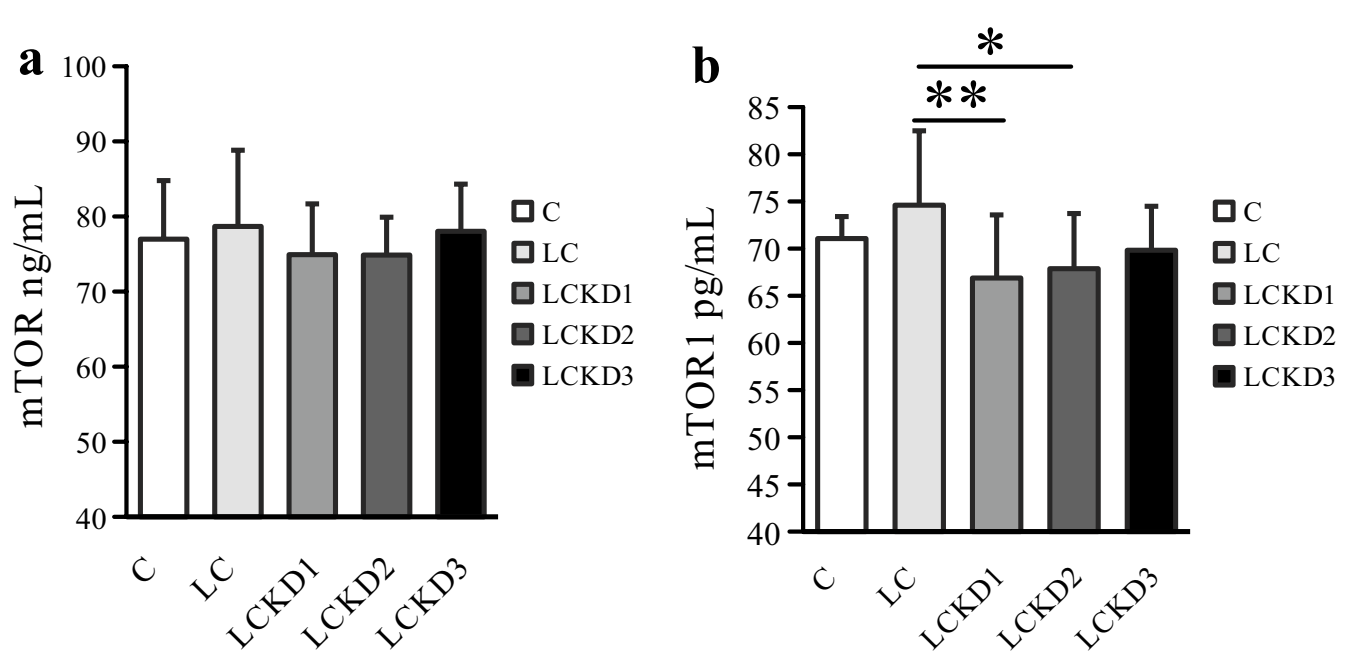

Fig. 5 Comparison of mTOR and mTOR1 levels in the rat serum in different groups. a The levels of mTOR; $\mathbf{b}$ the levels of mTOR1. Data are given as means \pm SD. Asterisks $\left(^{*}\right)$ represent significant differences between the two groups $\left(^{*} P<0.05 ;{ }^{* *} P<0.01\right.$ )
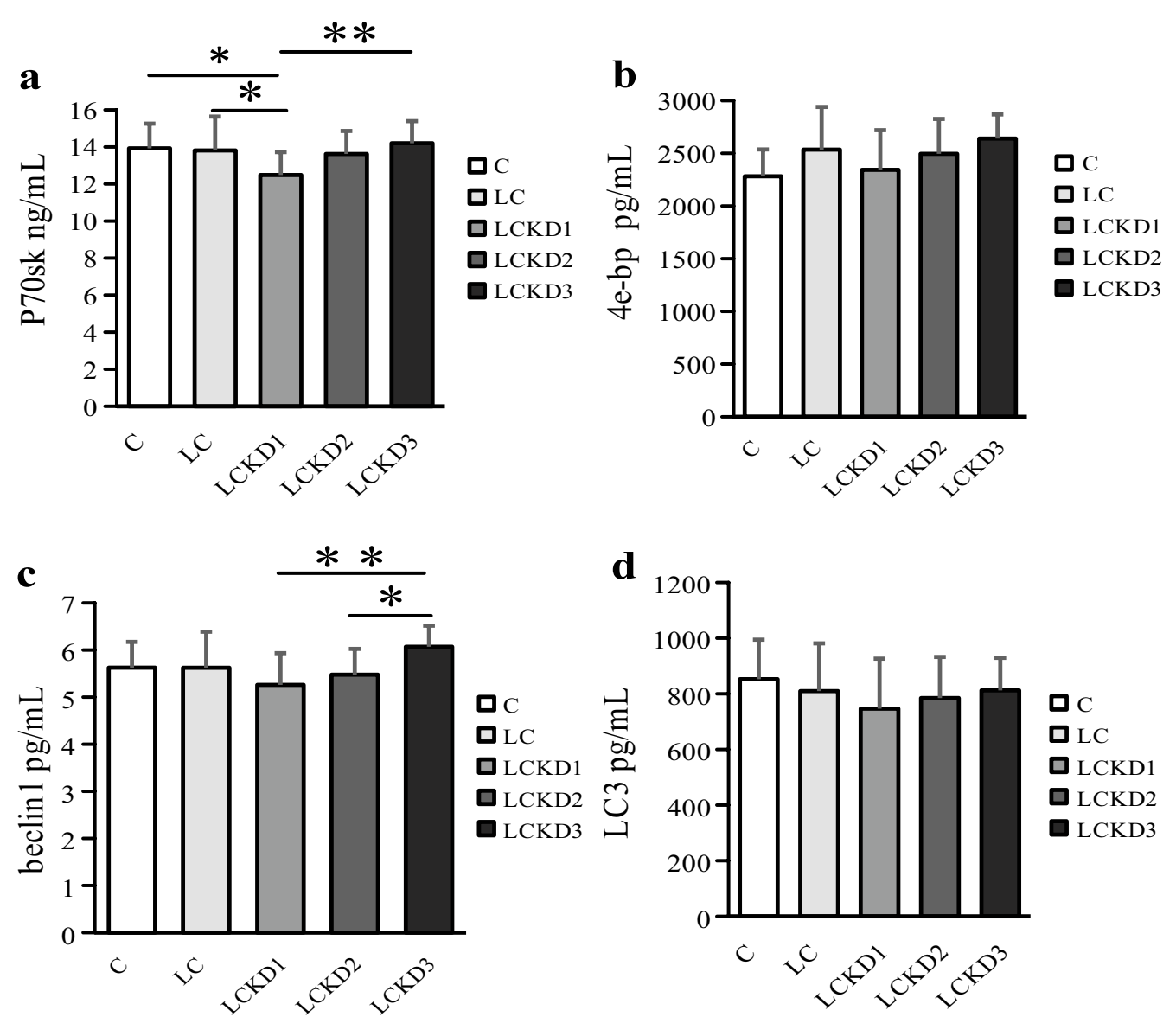

Fig. 6 Comparison of P70sk, 4e-bp, beclin-1 and LC-3 levels in the rat serum in different groups. a The levels of P70sk; $\mathbf{b}$ the levels of $4 \mathrm{e}-\mathrm{bp}$; $\mathbf{c}$ the levels of beclin-1; $\mathbf{d}$ the levels of LC-3. Data are given as means \pm SD. Asterisks $\left(^{*}\right)$ represent significant differences between the two groups $(*$ $P<0.05$; ** $P<0.01$ ) 
at 8-17 days, but the difference among the three groups was gradually decreased during the subsequent experimental period, which indicated that KD was effective on body weight in the early period, but the effect was slowly decreased in the later period. Notably, LCKD1 significantly reduced the weight of rats throughout the whole experiment. The only difference in feed composition of LCKD1 and LCKD2 were the MCFAs; therefore, the better effect on weight loss of LCKD1 can be attributed to medium-chain fatty acids, which was also confirmed by many researchers [21-23].

In order to reveal the mechanism of the difference on weight loss in rats treated by KD and MCFAs, blood biochemical parameters were determined. It can be seen from Fig. 1 that insulin levels were similar in all groups, whereas LC and KD significantly increased adiponectin. The results were consistent with previous study, which found the level of adiponectin was elevated under the stimulation of KD [24]. However, LCKD1 (containing MCFAs) had the opposite effect, suggesting that MCFAs could counteract the KD effect on adiponectin. This phenomenon was possibly because MCFAs were easier to oxidize and metabolize than long-chain fatty acids (LCFAs).

It is well known that ketogenic diet contains high fat, which can produce a large number of ketones. Unsurprisingly, the evident increase in blood ketone was observed in KD groups. Previous studies found that during the ketogenic period, subjects reported less hunger, but one of the putative causes of hunger may be due-together with other causes-to adiponectin [25-27]. Adiponectin was proved to be related to the hunger sensation, when it was bound to its receptor AdipoR1, AMP-activated protein kinase (AMPK) would be phosphorylated in the ARC of the hypothalamus [28]. LCKD1 (containing MCFAs) did not cause significant changes on adiponectin, as reported by another study [29], and they had the highest weight loss in rats at the end of the experiment. Previous studies from our group showed that a MCFAsrich diet led to a substantially greater increase in markers of mitochondrial content than LCFAs, and prevented the lipid accumulation and insulin resistance in this tissue [30]. Some studies reported that compared with LCT, MCT could increase energy expenditure and hepatic fatty acid oxidation in rats and humans [31-33]. Some results also showed that the oxidation and synthesis of fatty acids in rat white adipose tissue (WAT) were different when MCT and LCT were ingested [34]. Hormonesensitive lipase (HSL), considered as a potential target for the treatment of lipid disorders and obesity [35, 36], was believed to be the major enzyme responsible for the hydrolysis of stored triglycerides in adipose tissue. And some authors suggested that HSL, together with other yet unidentified lipases, may manipulate the mobilization of fatty acids in adipocytes. Thus, like HLS-cAMP, LCKD1 (containing MCFAs) may affect the weight of rats through other ways [37].

In order to investigate the effects on inflammatory factors as treated by MCFAs and KD, we determined the TNF- $\alpha$, hs-CPR, ENA-78 and IL-6 content in serum. Among them, the changes of TNF- $\alpha$ were relatively higher, which indicated MCFAs had some effects on TNF- $\alpha$. Similar results were reported before, which found that MCT could improve the inflammatory response of the small intestine after replacing some enteric preparations of $n-6$ fatty acids [38], and a putative receptor for MCFAs, G protein-coupled receptor 84 (GPR84, could influence the TNF- $\alpha$ levels [39].

Then we explored the aging-related pathway (TOR pathway). The LCKD1 group remarkably reduced the AMPK levels when compared with the other four groups, showing that MCFAs may affect the AMPK. Previous study proved that MCFAs could activate cAMP-dependent protein kinase (PKA) through HSL [37]. And because of the well-known relations between AMP/ATP and AMPK [40], it can be surmised that LCKD1 (containing MCFAs) might have an impact through AMP/ATP and AMPK pathway. Similarly, because TNF- $1 \alpha$ was found to be closely related to the inhibition of PI3K/Akt pathway, and MCFAs might activate PI3k pathway in cells [41], it was speculated that MCFAs could affect PI3K through TNF- $\alpha$ pathway.

Because the upstream pathway of mTOR was inhibited by KD and MCFAs, we determined the content of mTOR. The results showed that LCKD1 and LCKD2 groups reduced the mTOR1 level when compared with LC group. Many dietary interventions known to extend or modulate lifespan have been shown to be mediated, at least partially, by decreased mTORC1 activity [42], and one of the dietary interventions was KDs [43]. The effect of LCKD1 on mTOR was more obvious than that of LCKD2, which indicated a satisfying effect of MCFAs on mTORC1. LCKD1 also resulted in the reduction of the P70sk concentration in the downstream pathway of mTOR, suggesting that KD with MCFAs had an obvious effect on mTOR pathway.

As an effective treatment for intractable epilepsy, KD has not only traditional anticonvulsant effects, but also disease-modifying and antiepileptogenic properties in humans and animal models [18]. Rapamycin, an mTOR inhibitor, could prevent the development of epilepsy and the underlying pathophysiological mechanisms leading to epilepsy [43, 44]. Many studies reported that KD inhibited mTOR signaling pathway, but different components of KD caused different results [18]. We found that LCKD2 affected mTORC1 and its upstream pathway, 
but there was no significant change in the downstream, which was presumably caused by the KD composition and other experimental conditions such as rat size, location, etc. At the same time, there were no obvious changes in LC group in this experiment, probably due to the limited energy of only $20 \%$.

\section{Conclusions}

To summarize, KD and LC were effective in causing the weight loss of tested rats. KD without MCFAs could increase the content of ketone and adiponectin, and then affect the body weight, but little effect was detected on mTOR signaling pathway. With the addition MCFAs, however, it could not only increase the content of ketone in blood, reduce the inflammatory factor TNF-alpha, but also inhibit the mTOR pathway.

\begin{abstract}
Abbreviations
KD: Ketogenic diet; MCFAs: Medium-chain fatty acids; SD: Sprague-Dawley; C: Control diet; LC: 20\% Caloric restriction diet; LCKD1: 20\% Caloric restriction ketogenic diet; LCKD2: 20\% Caloric restriction ketogenic diet (containing MCFAs); LCKD3: 20\% Caloric restriction foreign ketogenic diet; MCT: Mediumchain triglycerides; PI3K: Phosphoinositide-3 kinase; AMPK: AMP-activated protein kinase; LCT: Long-chain fatty acids; LSD: Least significant difference; WAT: White adipose tissue; HSL: Hormone-sensitive lipase; GPR84: G proteincoupled receptor 84; PKA: CAMP-dependent protein kinase.
\end{abstract}

\section{Acknowledgements}

The authors thank Feng Li (Guangzhou ketone Co. Ltd) for the ketogenic products.

\section{Authors' contributions}

The authors' responsibilities were as follows- CL, FL, HL (Hui Li), QP: designed the research protocol; JH, HL (Huan Liu): conducted the research; HL (Huan Liu): analyzed the data, performed statistical analyses, and drafted the manuscript. All authors read and approved the final manuscript.

\section{Funding}

This work was supported by National Key Research and Development Program of Thirteenth Five-Year Plan (No. 2017YFC1601702) and Research and Development Program in Key Areas of Guangdong Province (No. 2019B020210002).

\section{Availability of data and materials}

Additional data may be availed on request to the authors through the corresponding author. We take legal responsibility for information, used procedures, data and results.

\section{Ethics approval and consent to participate}

This research work meets all the ethical guidelines, adhering to the legal requirements of my country.

\section{Consent for publication}

The authors confirm that there is no conflict of interest and agree with submission of the manuscript to your journal.

\section{Competing interests}

The authors declare that they have no competing interests.

\section{Author details}

${ }^{1}$ College of Food Science, Guangdong Provincial Key Laboratory of Food Quality and Safety, South China Agricultural University, Guangzhou 510642, China. ${ }^{2}$ Guangdong Testing Institute of Product Quality Supervision,
Foshan 528300, China. ${ }^{3}$ Guangzhou Ketone Co. Ltd, Guangzhou 510642, China. ${ }^{4}$ Logistics Department, South China Agricultural University, Guangzhou 510642, China.

Received: 11 July 2020 Accepted: 1 October 2020

Published online: 16 December 2020

\section{References}

1. Laux L, Blackford R. The ketogenic diet in Dravet syndrome. J Child Neurol. 2013;28:1041-4.

2. Korsholm K, Law I. Effects of a ketogenic diet on brain metabolism in epilepsy. Clin Nucl Med. 2013;38:38-9.

3. Auvin S. Should we routinely use modified Atkins diet instead of regular ketogenic diet to treat children with epilepsy? Seizure. 2012;21:237-40.

4. Paoli A, Rubini A, Volek JS, Grimaldi KA. Beyond weight loss: a review of the therapeutic uses of very-low-carbohydrate (ketogenic) diets. Eur J Clin Nutr. 2014:67:789-96.

5. Wilder RM. The effects of ketonemia on the course of epilepsy. Mayo Clin Proc. 1921;2:307-8.

6. Neal EG, Chaffe H, Schwartz RH, Lawson MS, Edwards N, Fitzsimmons $\mathrm{G}$, et al. The ketogenic diet for the treatment of childhood epilepsy: a randomised controlled trial. Lancet Neurol. 2008;7:500-6.

7. Freeman JM, Vining EP, Kossoff EH, Pyzik PL, Ye X, Goodman SN. A blinded, crossover study of the efficacy of the ketogenic diet. Epilepsia. 2009;50:322-5.

8. Kossoff EH, Zupec-Kania BA, Amark PE, Ballaban-Gil KR, Christina Bergqvist AG, Blackford R, Buchhalter JR, Caraballo RH, Cross JH, Dahlin MG, Donner EJ, Klepper J, Jehle RS, Kim HD, Christiana Liu YM, Nation J, Nordli DR, Pfeifer HH, Rho JM, Stafstrom CE, Thiele EA, Turner Z, Wirrell EC, Wheless JW, Veggiotti P, Vining EPG. Optimal clinical management of children receiving the ketogenic diet: recommendations of the International Ketogenic Diet Study Group. Epilepsia. 2009;50:304-17.

9. Barañano KW, Hartman AL. The ketogenic diet: uses in epilepsy and other neurologic illnesses. Curr Treat Options Neurol. 2008;10:410-9.

10. Huttenlocher PR, Wilbourne AJ, Sigmore JM. Medium chain triglycerides as a therapy for intractable childhood epilepsy. Neurology. 1971;21:1097-103.

11. Bach AC, Babayan VK. Medium-chain triglycerides: an update. Am J Clin Nutr. 1982;36:950-62.

12. Martin K, Jackson CF, Levy RG, Cooper PN. Ketogenic diet and other dietary treatments for epilepsy. Cochrane Database Syst Rev. 2016;9:2.

13. Chang P, Orabi B, Deranieh RM, Dham M, Hoeller O, Shimshoni JA, Yagen B, Bialer M, Greenberg ML, Walker MC, Williams RS. The antiepileptic drug valproic acid and other medium-chain fatty acids acutely reduce phosphoinositide levels independently of inositol in Dictyostelium. Dis Model Mech. 2012;5:115-24.

14. Hughes SD, Kanabus M, Anderson G, Hargreaves IP, Rutherford T, Donnell MO, Helen Cross J, Rahman S, Eaton S, Heales SJ. The ketogenic diet component decanoic acid increases mitochondrial citrate synthase and complex I activity in neuronal cells. J Neurochem. 2014;129:426-33.

15. Schönfeld P, Wojtczak L. Short-and medium-chain fatty acids in energy metabolism: the cellular perspective. J Lipid Res. 2016;57:943-54.

16. Shamji AF, Nghiem P, Schreiber SL. Integration of growth factor and nutrient signaling: implications for cancer biology. Mol Cell. 2003;12:271-80.

17. Dennis PB, Jaeschke A, Saitoh M, Fowler B, Kozma SC, Thomas G. Mammalian TOR: a homeostatic ATP sensor. Science. 2001;294:1102-5.

18. McDaniel SS, Rensing NR, Thio LL, Yamada KA, Wong M. The ketogenic diet inhibits the mammalian target of rapamycin (mTOR) pathway. Epilepsia. 2011;52:e7-e11.

19. Neshat MS, Mellinghoff IK, Tran C, Stiles B, Thomas G, Petersen R, Frost P, Gibbon JJS, Wu H, Sawyers CL. Enhanced sensitivity of PTENdeficient tumors to inhibition of FRAP/mTOR. Proc Natl Acad Sci USA. 2001;98:10314-9.

20. Huang Q, Ma S, Tominaga T, Suzuki K, Liu C. An 8-week, low carbohydrate, high fat, ketogenic diet enhanced exhaustive exercise capacity in mice part 2: effect on fatigue recovery, post-exercise biomarkers and antioxidation capacity. Nutrients. 2018;10:1339. 
21. St-Onge MP, Bosarge A. Weight-loss diet that includes consumption of medium-chain triacylglycerol oil leads to a greater rate of weight and fat mass loss than does olive oil. Am J Clin Nutr. 2008;87:621-6.

22. Montgomery MK, Osborne B, Brown SH, Small L, Mitchell TW, Cooney GJ, Turner N. Contrasting metabolic effects of medium-versus long-chain fatty acids in skeletal muscle. J Lipid Res. 2013;54:3322-33.

23. Liu WL, Liu W, Liu CM, Yang SB, Liu JH, Zheng HJ, Su KM. Medium-chain fatty acid nanoliposomes suppress body fat accumulation in mice. $\mathrm{Br} J$ Nutr. 2011:106:1330-6.

24. Monda V, Polito R, Lovino A, Finaldi A, Valenzano A, Nigro E, Corso G, Sessa F, Asmundo A, Nunno ND, Cibelli G, Messina G. Short-term physiological effects of a very low-calorie ketogenic diet: effects on adiponectin levels and inflammatory states. Int J Mol Sci. 2020;21:3228.

25. Nickols-Richardson SM, Coleman MD, Volpe JJ, Hosig KW. Perceived hunger is lower and weight loss is greater in overweight premenopausal women consuming a low-carbohydrate/high-protein vs high-carbohydrate/low-fat diet. J Am Diet Assoc. 2005;105:1433-7.

26. Johnston CS, Tjonn SL, Swan PD, White A, Hutchins H, Sears B. Ketogenic low-carbohydrate diets have no metabolic advantage over nonketogenic low-carbohydrate diets. Am J Clin Nutr. 2006;83:1055-61.

27. Johnstone AM, Horgan GW, Murison SD, Bremner DM, Lobley GE. Effects of a high-protein ketogenic diet on hunger, appetite, and weight loss in obese men feeding ad libitum. Am J Clin Nutr. 2008;87:44-55.

28. Paoli A, Bosco G, Camporesi EM, Mangar D. Ketosis, ketogenic diet and food intake control: a complex relationship. Front Psychol. 2015;6:27.

29. Nagasaki H, Kondo T, Fuchigami M, Hashimoto H, Sugimura Y, Ozaki N, Arima H, Ota A, Oiso Y, Hamada Y. Inflammatory changes in adipose tissue enhance expression of GPR84, a medium-chain fatty acid receptor:TNFa enhances GPR84 expression in adipocytes. FEBS Lett. 2012;586:368-72.

30. Turner N, Hariharan K, TidAng J, Frangioudakis G, Beale SM, Wright LE, Zeng XY, Leslie SJ, Li JY, Kraegen EW, Cooney GJ, Ye J. Enhancement of muscle mitochondrial oxidative capacity and alterations in insulin action are lipid species dependent: potent tissue-specific effects of mediumchain fatty acids. Diabetes. 2009;58:2547-54.

31. Shinohara H, Ogawa A, Kasai M, Aoyama T. Effect of randomly interesterified triacylglycerols containing medium-and long-chain fatty acids on energy expenditure and hepatic fatty acid metabolism in rats. Biosci Biotechnol Biochem. 2005;69:1811-8.

32. St-Onge MP, Ross R, Parsons WD, Jones PJ. Medium-chain triglycerides increase energy expenditure and decrease adiposity in overweight men. Obes Res. 2003;11:395-402.

33. Alexandrou E, Herzberg GR, White MD. High-level medium-chain triglyceride feeding and energy expenditure in normal-weight women. Can J Physiol Pharmacol. 2007;85:507-13.
34. Shinohara $\mathrm{H}, \mathrm{Wu} J$, Kasai M, Aoyama T. Randomly interesterified triacylglycerol containing medium- and long-chain fatty acids stimulates fatty acid metabolism in white adipose tissue of rats. Biosci Biotechnol Biochem. 2006;70:2919-26.

35. Large V, Reynisdottir S, Langin D, Fredby K, Klannemark M, Holm C, Arner P. Decreased expression and function of adipocyte hormonesensitive lipase in subcutaneous fat cells of obese subjects. J Lipid Res. 1999:40:2059-65

36. Haemmerle G, Zimmermann R, Zechner R. Letting lipids go: hormonesensitive lipase. Curr Opin Lipidol. 2003;14:289-97.

37. Liu Y, Xue C, Zhang Y, Xu Q, Yu X, Zhang X, Wang J, Zhang R, Gang X, Guo $C$. Triglyceride with medium-chain fatty acids increases the activity and expression of hormone-sensitive lipase in white adipose tissue of C57BL/6J mice. Biosci Biotechnol Biochem. 2011;75:1939-44.

38. Mañé J, Pedrosa W, Lorén V, Ojanguren I, Fluvià L, Cabré E, Rogler G, Gassull MA. Partial replacement of dietary (n-6) fatty acids with mediumchain triglycerides decreases the incidence of spontaneous colitis in Interleukin-10 deficient Mice. J Nutr. 2009;139:603-10.

39. Suzuki M, Takaishi S, Nagasaki M, Onozawa Y, lino I, Maeda H, Komai T, Oda T. Medium-chain fatty acid-sensing receptor, GPR84, is a proinflammatory receptor. J Biol Chem. 2013;288:10684-91.

40. Dasgupta B, Chhipa RR. Evolving lessons on the complex role of AMPK in normal physiology and cancer. Trends Pharmacol Sci. 2015;37:192-206.

41. Kamata Y, Shiraga H, Tai A, Kawamoto Y, Gohda E. Induction of neurite outgrowth in PC12 cells by the medium-chain fatty acid octanoic acid. Neuroscience. 2007;146:1073-81.

42. Houtkooper RH, Williams RW, Auwerx J. Metabolic networks of longevity. Cell. 2010;142(1):9-14

43. Zeng LH, Xu L, Gutmann DH, Wong M. Rapamycin prevents epilepsy in a mouse model of tuberous sclerosis complex. Ann Neurol. 2008:63:444-53.

44. Zeng LH, Rensing NR, Wong M. The mammalian target of rapamycin signaling pathway mediates epileptogenesis in a model of temporal lobe epilepsy. J Neurosci. 2009;29:6964-72.

\section{Publisher's Note}

Springer Nature remains neutral with regard to jurisdictional claims in published maps and institutional affiliations.

\section{Submit your manuscript to a SpringerOpen ${ }^{\circ}$ journal and benefit from:}

- Convenient online submission

- Rigorous peer review

- Open access: articles freely available online

- High visibility within the field

Retaining the copyright to your article

Submit your next manuscript at springeropen.com 